\title{
Is abdominal compression useful in lung stereotactic body radiation therapy? A 4DCT and dosimetric lobe-dependent study
}

\author{
Gauthier Bouilhol ${ }^{\text {a,b,*}}$, Myriam Ayadi ${ }^{\text {b }}$, Simon Rit ${ }^{\text {a,b }}$, \\ Sheeba Thengumpallil ${ }^{b}$, Joël Schaerer ${ }^{a, b}$, \\ Jef Vandemeulebroucke ${ }^{c, d}$, Line Claude ${ }^{b}$, David Sarrut ${ }^{a, b}$
}

\author{
a University of Lyon, CREATIS, CNRS UMR 5220, Inserm U1044, INSA-Lyon, Université Lyon 1, \\ Villeurbanne, France \\ ${ }^{\mathrm{b}}$ Department of Radiation Oncology, Léon Bérard Cancer Center, Lyon, France \\ ${ }^{c}$ Vrije Universiteit Brussel (VUB), Dept. of Electronics and Informatics (ETRO), Pleinlaan 2, \\ B-1050 Brussels, Belgium \\ ${ }^{\mathrm{d}}$ Interdisciplinary Institute for Broadband Technology (IBBT), Dept. of Future Media and \\ Imaging (FMI), Gaston Crommenlaan 8 (box 102), B-9050 Ghent, Belgium
}

Received 18 January 2012; received in revised form 8 April 2012; accepted 24 April 2012

Available online 20 May 2012

\section{KEYWORDS \\ Lung SBRT; \\ Abdominal \\ compression; \\ Motion reduction}

\begin{abstract}
Purpose: To determine the usefulness of abdominal compression in lung stereotactic body radiation therapy (SBRT) depending on lobe tumor location.

Materials and methods: Twenty-seven non-small cell lung cancer patients were immobilized in the Stereotactic Body Frame ${ }^{\mathrm{TM}}$ (Elekta). Eighteen tumors were located in an upper lobe, one in the middle lobe and nine in a lower lobe (one patient had two lesions). All patients underwent two four-dimensional computed tomography (4DCT) scans, with and without abdominal compression. Three-dimensional tumor motion amplitude was determined using manual landmark annotation. We also determined the internal target volume (ITV) and the influence of abdominal compression on lung dose-volume histograms.

Results: The mean reduction of tumor motion amplitude was $3.5 \mathrm{~mm}(p=0.009)$ for lower lobe tumors and $0.8 \mathrm{~mm}(p=0.026)$ for upper/middle lobe locations. Compression increased tumor motion in 5 cases. Mean ITV reduction was $3.6 \mathrm{~cm}^{3}(p=0.039)$ for lower lobe and $0.2 \mathrm{~cm}^{3}(p=0.048)$ for upper/middle lobe lesions. Dosimetric gain of the compression for lung sparing was not clinically relevant.
\end{abstract}

\footnotetext{
* Corresponding author. Centre de Lutte Contre le Cancer Léon Bérard, Service de Radiothérapie-Physique, 28 rue Laennec, 69008 Lyon, France. Tel.: +3347878 51 51; fax: +33478785998.

E-mail address: bouilhol@creatis.insa-lyon.fr (G. Bouilhol).
} 
Conclusions: The most significant impact of abdominal compression was obtained in patients with lower lobe tumors. However, minor or negative effects of compression were reported for other patients and lung sparing was not substantially improved. At our institute, patients with upper or middle lobe lesions are now systematically treated without compression and the usefulness of compression for lower lobe tumors is evaluated on an individual basis.

(c) 2012 Associazione Italiana di Fisica Medica. Published by Elsevier Ltd. All rights reserved.

\section{Introduction}

Conventional radiation therapy for medically inoperable early stage non-small cell lung cancers (NSCLC) is associated with poor local control and survival rate [1]. Stereotactic body radiation therapy (SBRT) is an effective alternative for malignancies like these as it substantially improves treatment outcomes [2-6]. SBRT consists in the delivery of ablative radiation doses in hypofractionated schedules with 5 fractions or less in order to improve tumor local control up to $90 \%$ with biologically equivalent doses that can exceed $150 \mathrm{~Gy}$ [7]. To achieve acceptable normal tissue exposure, small T1-T2NOMO peripheral tumors with a diameter smaller than $5 \mathrm{~cm}$ and a distance to the proximal bronchial tree greater than $2 \mathrm{~cm}$ are typically treated with SBRT $[8,9]$. In practice, the delivery of such high doses per fraction requires very high setup accuracy and robust motion management, with highly conformal beam arrangements and small safety margins.

Immobilization of the patient can be enforced with commercially available devices [10], e.g. stereotactic frames [11,12] or vacuum systems [13]. Accurate knowledge of the position and shape of the gross tumor volume (GTV) during the breathing cycle is essential and assessment by four-dimensional computed tomography (4DCT) is recommended [14]. A common irradiation strategy consists in irradiating the internal target volume (ITV), corresponding to the volume covered by the moving GTV. The maximum intensity projection (MIP) images derived from the 4DCT dataset can be used to delineate the ITV [15].

An abdominal compression device can be added to the stereotactic frame to reduce tumor motion and associated margins $[16,17]$. However, it may cause discomfort with possible anxiety for some patients, being a potential source of increase of tumor motion variability according to Bissonnette et al. [18]. Moreover, it is unusable for obese patients, and at our institute we estimate that this technique increases treatment preparation time by about 45 min (see Patient immobilization and CT scan acquisitions sections for more details) and daily in-room time by about 10 min in comparison to conventional treatments.

The efficiency of abdominal compression for reducing lung tumor motion has been assessed in several studies [19-22]. These studies did not give three-dimensional results [19] according to lobe tumor location [19-21] or reported results substantially different from ours [22]. The dosimetric impact of abdominal compression on irradiated lung tissue has been investigated by Kontrisova et al. [23] but results were not given according to lobe tumor location.

The present retrospective study aimed at evaluating the relevance of abdominal compression in 27 SBRT patients, depending on tumor location within the lung. We first quantified the influence of abdominal compression on geometrical parameters. The efficiency of abdominal compression for reducing three-dimensional tumor motion and the ITV was evaluated on 4DCT images acquired with and without compression. The second part of the study aimed at quantifying the influence of abdominal compression on dosimetric parameters in order to estimate the possible improvement in lung toxicity. The measurements and calculations presented in this paper systematically took into account motion artifacts in 4DCT images (see description of Geometrical study).

\section{Materials and methods}

\section{Patient and tumor characteristics}

Twenty-seven peripheral NSCLC patients who underwent lung SBRT were included in this study, corresponding to twenty-eight tumors (one patient had two lung lesions). Eighteen tumors were located in the upper lobe, one in the middle lobe and nine in the lower lobe. The patients were divided in two groups: lower lobe and upper/middle lobe. This distinction was made considering that diaphragmatic motion is higher in its posterior region, where the lower lobe is located, in contrast to the upper lobe and the middle lobe.

\section{Patient immobilization}

All patients were installed in the Stereotactic Body Frame ${ }^{\mathrm{TM}}$ (SBF, Elekta AB, Stockholm, Sweden) associated to the abdominal compression plate. The compression level was then adjusted under fluoroscopy using the in-room conebeam CT system to reduce residual diaphragmatic motion amplitude below $1 \mathrm{~cm}$ in the cranio-caudal direction [24].

\section{CT scan acquisitions}

Patients underwent two helical 4DCT scans using the Brilliance Big Bore ${ }^{\mathrm{TM}}$ (Philips) CT simulation system. The first scan was performed without compression and the second one with pre-determined compression. CT scans were performed with a 0.5 s rotation period and a 0.1 pitch. The beam collimation width was $24 \mathrm{~mm}(16 \times 1.5 \mathrm{~mm})$ and breathing synchronization was achieved with the Pneumo Chest Bellows ${ }^{\mathrm{TM}}$ (Lafayette Instrument). Ten phases, the MIP images [25] and the untagged $C T$ sets were reconstructed from the two 4DCT scans. The untagged images resulted from the 3D tomographic reconstruction using all data, i.e. without any respiration-correlated data binning. In this way, blurred images are obtained, reflecting the average density of the structures during the breathing cycle. The voxel size was $1.17 \times 1.17 \times 2 \mathrm{~mm}^{3}$ (where $2 \mathrm{~mm}$ is the slice thickness). 


\section{Geometrical study}

The geometrical study was performed with home-made open-source software dedicated to $4 \mathrm{D}$ visualization and image processing [26,27]. In previous studies [20,21], tumor motion was assessed by measuring the displacement of its centre of mass. However the position of the centre of mass depends on the presence of motion artifacts in the tumor area. The present method consisted in measuring threedimensional tumor motion amplitude on the 4DCT datasets with manual landmark annotation (tumor deformation was overlooked). The chosen landmarks were particularities of the tumor surface identifiable in a reproducible way from one extreme phase of the respiratory cycle to the other and from one acquisition to the other (with/without compression). The method consisted in displaying simultaneously the four images corresponding to end-exhale and end-inhale phases, with and without compression. For each acquisition, the tumor extreme phases were preliminarily determined by visualizing the complete $4 D C T$ dataset. Then, we identified the corresponding slices of the tumor on the four images. The same recognizable voxel was selected by progressive zooming (Fig. 1). Method and images used for image annotation were similar to those used by Vandemeulebroucke et al. [28] who evaluated the inter-observer variability with the mean distance between annotations at $0.5 \mathrm{~mm}$ (SD $0.9 \mathrm{~mm}$ ). To account for motion artifacts in the tumor region and ensure the consistency of the measurement method, landmarks were chosen in order to reflect the largest tumor motion amplitude at the extreme phases, representing the tumor motion amplitude closest to the maximum tumor excursion. Figure 2 shows an example of landmark placement in case of an artifact in an end-inhale image. In this case, the tumor is supposed to be at its lowest position.

The modification of the tumor motion amplitude provides no volumetric information because this depends

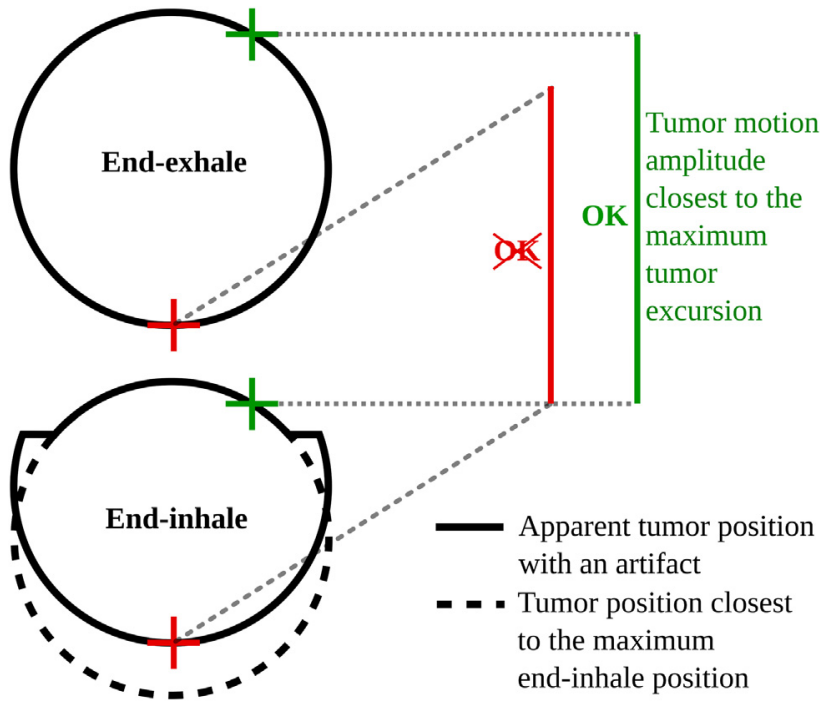

Figure 2 Illustration of the landmark registration method with an artifact in the tumor region in an end-inhale phase: selection of a voxel representing the highest tumor motion amplitude.

on the tumor size. We studied the influence of abdominal compression on the ITV. Problems induced by motion artifacts can lead to errors in the estimation of the ITV if measured from the MIP volume. This way, differences in ITV size between images acquired with and without compression can be missed or falsely detected. For this reason, a theoretical study based on the previous landmark registration was performed. The goal was to form a global idea of how the ITV is influenced by the use of compression. We considered each tumor as a sphere moving along a straight line from one extreme phase to the other. The ITV is then given by the following formula:

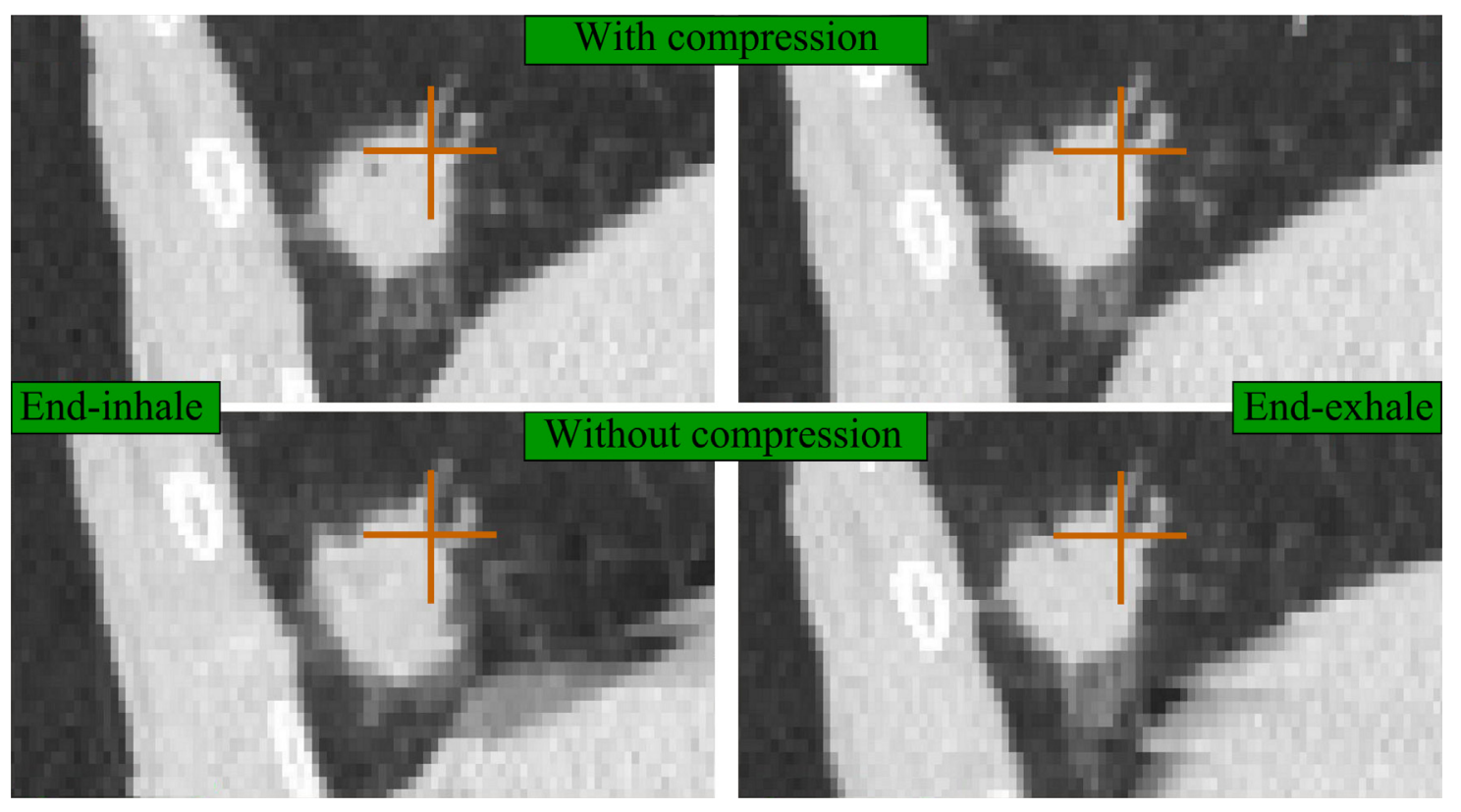

Figure 1 Landmark registration between the four 4DCT phases (end-exhale and end-inhale, with and without compression). Selection of the corresponding slices on the four images in the tumor region and selection of the same recognizable voxel. 
$\mathrm{ITV} \approx \frac{4}{3} \pi r^{3}+\pi r^{2} A_{3 D}$

where $r$ is the tumor radius and $A_{3 D}$ the overall motion amplitude between extreme phases.

Paired $t$-tests between geometrical results obtained with and without abdominal compression were performed in order to quantify the statistical significance of the gain obtained with abdominal compression.

\section{Dosimetric study}

As observed by Yamamoto et al. [29], most 4DCT images from the 27 patients contained artifacts. Dosimetric calculation using images with artifacts cannot provide relevant results, particularly in case of artifacts in the tumor region or in critical structures as mentioned in the report of AAPM Task Group 101 [10]. Indeed, motion artifacts can result in errors in ITV delineation and in lung delineation. In addition, motion artifacts impact differently images acquired with and without abdominal compression (see Discussion section for more details) so that it becomes difficult to attribute dosimetric gains to the use of compression or to motion artifacts. Therefore, only patients without artifacts seriously impacting the ITV or the lung volume were selected for the study. Moreover, among these patients we selected those with the highest geometrical efficiency based on the geometrical study, resulting in the selection of three lower lobe patients. This enabled us to focus on images for which the highest dosimetric efficiency of abdominal compression was expected. An upper lobe patient with artifact-free images was also chosen to extend the study to another location in the lung. Thus four of the 27 patients initially included were selected (see Figs. 3 and 4 for more details).

The dosimetric study consisted of assessing the impact of abdominal compression in terms of dose received by the healthy lung tissue. Treatment planning was performed on untagged images. The ITV was delineated (FOCAL ${ }^{\mathrm{TM}}, \mathrm{CMS}$ ) on the 4DCT MIP reconstruction and the lungs were delineated on the untagged images. The planning target volume (PTV) resulted from the addition of a $8 \mathrm{~mm}$ margin around the ITV in the cranio-caudal direction and $5 \mathrm{~mm}$ in the leftright and antero-posterior directions. The treatment plan consisted of 12 static coplanar fields of $6 \mathrm{MV}$ photon beams. The fields were shaped to the PTV without additional margins, equally-distributed around the patient and equally-weighted. A dose of 48 Gy (4 fractions) was prescribed to the $80 \%$ isodose line. We made sure that at least $95 \%$ of the PTV was conformally covered by the prescription isodose line [9] and the coverage was similar in the two CT scans (with and without abdominal compression). Dose calculation was performed on a $\mathrm{XiO}^{\mathrm{TM}}$ workstation (CMS) using a superposition algorithm and a $2 \mathrm{~mm}$ calculation grid. Then we analyzed the lung dose-volume histograms (DVH). We examined the influence of abdominal compression on the lung volume receiving $16 \mathrm{~Gy}\left(V_{16}\right)$ and $20 \mathrm{~Gy}\left(V_{20}\right)$, on the dose received by $1000 \mathrm{~cm}^{3}\left(D_{1000}\right)$ and $1500 \mathrm{~cm}^{3}\left(D_{1500}\right)$ of lung and on the mean lung dose $\left(D_{\text {mean }}\right)$, since all these parameters have been reported to be predictive of lung toxicity [9].

\section{Results}

\section{Geometrical efficiency}

Free (i.e. without abdominal compression) tumor motion amplitude was $12.4 \mathrm{~mm}$ in average (SD $6.5 \mathrm{~mm}$, range 2.6-24.7 mm) for tumors in the lower lobe and $4.9 \mathrm{~mm}$ (SD $3.8 \mathrm{~mm}$, range $1.2-14.1 \mathrm{~mm}$ ) for the upper/middle lobe. When applying the compression, mean tumor motion amplitude was reduced to $8.9 \mathrm{~mm}$ (SD $4.2 \mathrm{~mm}$, range 1.7-16.4 mm) for lower lobe tumors and $4.1 \mathrm{~mm}$ (SD $3.4 \mathrm{~mm}$, range $0.0-13.1 \mathrm{~mm}$ ). Figure 3 shows modifications of tumor motion amplitude using abdominal compression. Results are sorted by cranio-caudal position, distinguishing between lower and upper/middle lobe locations. Overall,
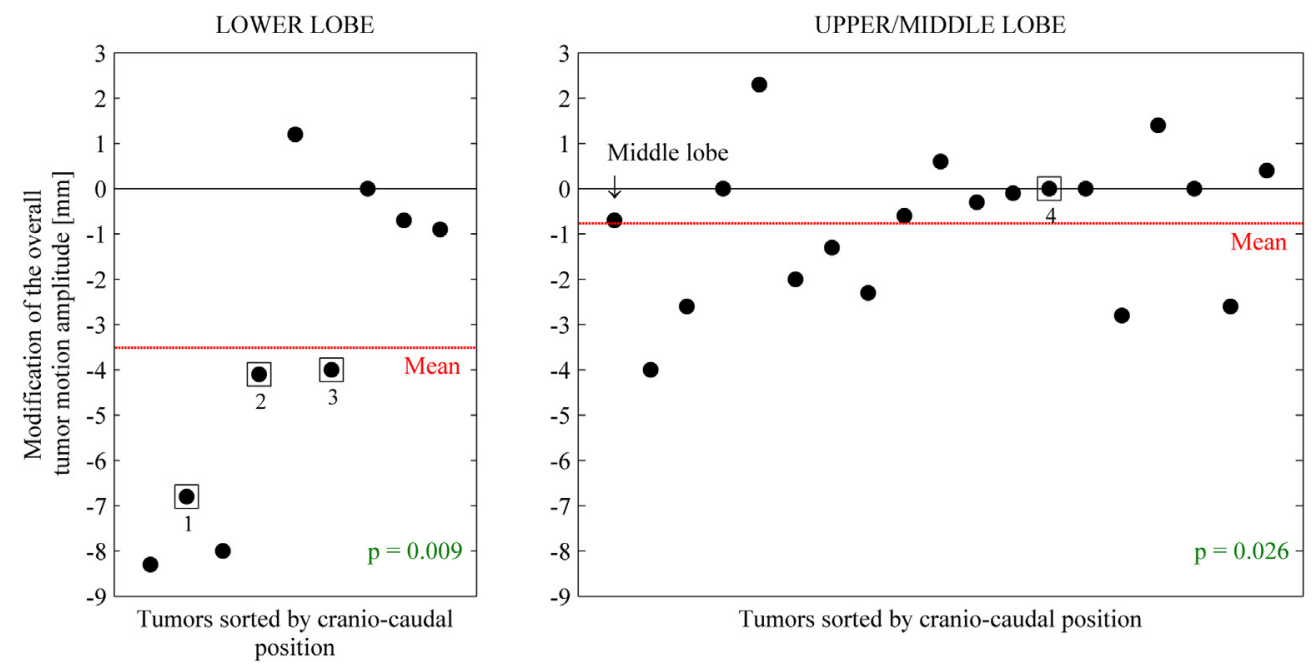

Figure 3 Modification of the overall tumor motion amplitude in mm. Negative (positive) values represent decreased (increased) tumor motion amplitudes due to the use of abdominal compression. Boxed results correspond to patients selected for the dosimetric study. Also presented are the $p$ values concerning the difference between the data obtained with and without abdominal compression. 

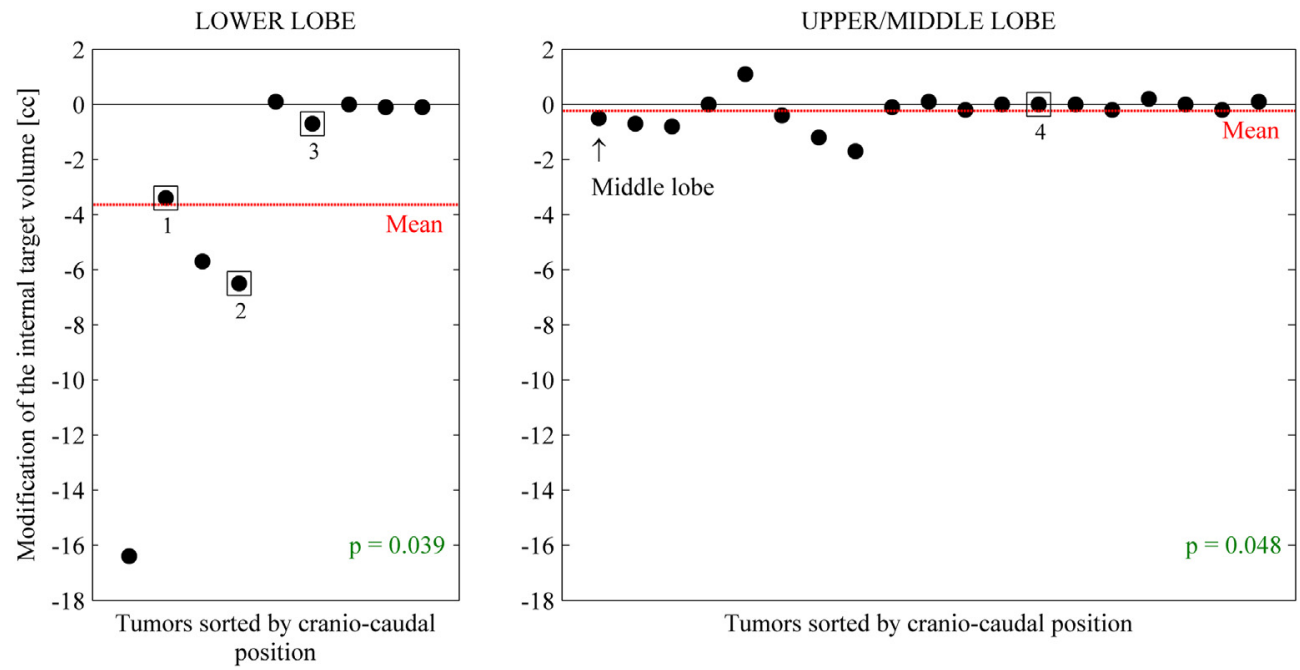

Figure 4 Modification of the internal target volume in $\mathrm{cm}^{3}$ depending on tumor location. Negative (positive) values represent decreased (increased) internal target volumes due to the use of abdominal compression. Boxed results correspond to patients selected for the dosimetric study. Also presented are the $p$ values concerning the difference between the data obtained with and without abdominal compression.

tumor motion was found to be significantly reduced $(p=0.002)$ in the 28 tumors involved in the study, with a mean reduction of $1.7 \mathrm{~mm}$. However, the results showed a variable influence of abdominal compression. A substantial reduction $(>3 \mathrm{~mm}$ ) was observed for 6 of the 28 tumors, a null or minor reduction $(<3 \mathrm{~mm})$ was obtained in 17 tumors, whereas for the 5 remaining patients the compression slightly increased tumor motion amplitude (maximum observed increase of $2.3 \mathrm{~mm}$ ).

The most significant benefit of abdominal compression was obtained in lower lobe tumors $(p=0.009)$ with a mean amplitude reduction of $3.5 \mathrm{~mm}$ (SD $3.6 \mathrm{~mm}$, range $1.2-8.3 \mathrm{~mm}$ ). The compression had a significant but limited influence for upper/middle lobe locations $(p=0.026)$ with a mean reduction of $0.8 \mathrm{~mm}$ (SD $1.6 \mathrm{~mm}$, range $-2.3-4.0 \mathrm{~mm}$ ). Negative effects resulting in a motion amplitude increase could be observed independently of tumor location.

Mean tumor size, corresponding to the largest tumor dimension, was $2.4 \mathrm{~cm}$ (SD $1.5 \mathrm{~cm}$, range $1-5 \mathrm{~cm}$ ) for the lower lobe and $2.1 \mathrm{~cm}$ (SD $0.9 \mathrm{~cm}$, range $1-4 \mathrm{~cm}$ ) for the upper/middle lobe. Figure 4 presents the influence of abdominal compression on the ITV analyzed using the theoretical approach described in Geometrical study. A notable reduction $\left(>2 \mathrm{~cm}^{3}\right)$ of the ITV was observed for 4 patients, all with lower lobe tumors. A mean reduction of $3.6 \mathrm{~cm}^{3}$ (SD $5.4 \mathrm{~cm}^{3}$, range $-0.1-16.3 \mathrm{~cm}^{3}$ ) was obtained for lower lobe lesions $(p=0.039)$ and of $0.2 \mathrm{~cm}^{3}$ (SD $0.6 \mathrm{~cm}^{3}$, range $-1.1-1.6 \mathrm{~cm}^{3}$ ) for upper/middle lobe lesions $(p=0.048)$. The overall mean ITV reduction was $1.3 \mathrm{~cm}^{3}$ in the 28 lesions and the influence of abdominal compression on the ITV was less significant $(p=0.024)$ than variations in tumor motion amplitude.

\section{Dosimetric benefit}

Absolute dosimetric gains are presented in Table 1. The mean relative modifications of dosimetric parameters were
$-7 \%,-5 \%,-5 \%,-14 \%$ and $-0 \%$ for $V_{16}, V_{20}, D_{1000}, D_{1500}$ and $D_{\text {mean }}$, respectively. The maximum relative gains were observed for lower lobe patients with modifications of $-16 \%$ (patient 1), $-19 \%$ (patient 1), $-19 \%$ (patient 2), $-33 \%$ (patient 3) and $-15 \%$ (patient 3) for $V_{16}, V_{20}, D_{1000}, D_{1500}$ and $D_{\text {mean }}$, respectively. A degradation of dosimetric parameters could also be observed with the use of abdominal compression for patient 3 with modifications of $+8 \%,+25 \%$ and $+15 \%$ for $V_{20}, D_{1000}$ and $D_{\text {mean }}$, respectively.

\section{Discussion}

Abdominal compression systematically reduces motion amplitude for tumors close to the diaphragm but it can have very different effects in other locations. Globally, the benefit on middle and upper lobe tumor motion is very limited whereas the gain can be important but is not systematic in lower lobe locations. An increase of motion amplitude could even be observed for $18 \%$ of the tumors, independently of the location. It may be due to the fact that, under abdominal compression, patients tend to breathe with the upper thoracic region, the lower part being immobilized. In addition, abdominal compression can increase the variability of tumor motion because of patient discomfort and anxiety [18].

Table 2 summarizes results reported by previous studies [19-22]. We obtained a significant mean reduction of $3.5 \mathrm{~mm}$ for lower lobe tumors $(p=0.009)$. Heinzerling et al. [20] have reported higher results with a significant mean motion reduction of 5.3 and $6.4 \mathrm{~mm}(p<0.0001)$ with two compression levels. The difference can be attributed to the fact that motion of both liver and lower lung lobe tumors was analyzed in this study. This suggests that abdominal compression is more efficient in reducing tumor motion in the liver than in the lower lung lobes. It is in accordance with Wunderink et al. [30] who have reported high efficiency of abdominal compression in reducing liver tumor motion. Concerning overall tumor motion amplitude 
Table 1 Absolute modifications of lung dosimetric parameters due to the use of abdominal compression according to tumor location: lower lobe (LL) and upper/middle lobe (UML).

\begin{tabular}{|c|c|c|c|c|c|c|c|}
\hline & Patient (location) & & 1 (LL) & 2 (LL) & 3 (LL) & 4 (UML) & Mean \\
\hline \multirow{2}{*}{$\begin{array}{l}\text { Volumetric } \\
\text { parameters }\end{array}$} & \multicolumn{2}{|c|}{ PTV (cc) } & -13.01 & -9.46 & -1.82 & -6.19 & -7.62 \\
\hline & \multicolumn{2}{|c|}{ Lungs (Left + Right - PTV) (cc) } & -131.01 & -164.57 & $-107.07^{a}$ & -153.89 & -139.13 \\
\hline \multirow{5}{*}{$\begin{array}{l}\text { Dosimetric } \\
\text { parameters }\end{array}$} & Lungs (Left + Right - PTV) & $V_{16}(\%)$ & -0.82 & -0.87 & -0.06 & -0.24 & -0.50 \\
\hline & & $V_{20}(\%)$ & -0.63 & -0.42 & +0.17 & -0.24 & -0.28 \\
\hline & & $D_{1000}(G y)$ & -0.65 & -1.80 & +0.42 & -0.16 & -0.55 \\
\hline & & $D_{1500}(\mathrm{~Gy})$ & -0.11 & -0.57 & -0.2 & -0.05 & -0.23 \\
\hline & & $D_{\text {mean }}(G y)$ & -0.29 & -0.33 & +0.54 & -0.05 & -0.03 \\
\hline
\end{tabular}

${ }^{\text {a }}$ Data available for the left lung only because this patient had a pulmonectomy.

in the whole lung, our results $(1.7 \mathrm{~mm}$ mean reduction, $p=0.002$ ) compared favorably with those of Han et al. [21] $(1.3 \mathrm{~mm}, p<0.05)$. The comparison of our results with those of Negoro et al. [19] was performed by restricting our analysis to cranio-caudal tumor displacements and to tumors with motion amplitude greater than $5 \mathrm{~mm}$ (10 patients). We found that abdominal compression significantly reduces tumor motion $(p=0.005)$ from a range of 6-24 mm (mean $13.0 \mathrm{~mm}$ ) to a range of 6-16 mm (mean $9.6 \mathrm{~mm}$ ). These results are in agreement with those of Negoro et al. [19] who have reported a significant reduction $(p=0.0002)$ from a range of $8-20 \mathrm{~mm}$ (mean $12.3 \mathrm{~mm})$ to a range of $2-11 \mathrm{~mm}$ (mean $7.0 \mathrm{~mm}$ ). Focusing on craniocaudal direction to compare with Bengua et al. [22], we obtained a significant mean amplitude reduction of $3.6 \mathrm{~mm}$ for lower lobe tumors $(p=0.006)$ and $0.2 \mathrm{~mm}$ for upper/ middle lobe tumors $(p=0.007)$. These results are substantially different from those of Bengua et al. [22] who have obtained no significant motion reduction with a mean of $0.48 \mathrm{~mm}$ for middle or lower lobe markers $(p=0.65)$ and $0.46 \mathrm{~mm}$ for upper lobe markers $(p=0.61)$. This difference may stem from the fact that the patients involved in their study were able to monitor their breathing amplitude from a respiration range indicator. Moreover, the gold markers used for tumor tracking were not systematically placed inside the tumor. However, one must keep in mind that the differences observed between the present study and literature may also be attributed to the use of different compression levels [20].

Results are more manifest regarding the influence of compression on the ITV. Indeed, an important benefit in tumor motion amplitude is necessary but not sufficient to obtain a notable volumetric gain. The reduction in motion must apply to large enough tumors to be really beneficial, but large tumors are not frequent in SBRT patients. To overcome problems in the measurement of the ITV in presence of motion artifacts in the tumor region, the modification of the ITV has been assessed using the landmark annotations on the tumor extreme phases and making the assumption of a one-dimensional displacement between them. This assumption does not account for potential tumor motion hysteresis $[31,32]$ but the aim was just to emphasize the fact that the reduction of the ITV is limited by the small size of the tumors treated with SBRT.

In spite of observable gains in ITV and PTV, the benefit of abdominal compression on lung dosimetric parameters appeared to be limited. The reason is that the compression not only reduces the target volume, but also has the systematic effect of reducing the lung volume (as observed by Kontrisova et al. [23]). Therefore, the proportion of the

Table 2 Previous studies on efficiency of abdominal compression associated with the SBF for reducing lung tumor motion.

\begin{tabular}{|c|c|c|c|c|c|c|}
\hline Authors & Year & $N$ & Location & Modality & Direction & Results \\
\hline $\begin{array}{l}\text { Negoro } \\
\text { et al. [19] }\end{array}$ & 2001 & 10 & - & Fluoroscopy & $\mathrm{CC}$ & $\begin{array}{l}\text { Reduction from a range } \\
\text { of } 8-20 \mathrm{~mm} \text { (mean } 12.3 \mathrm{~mm}) \\
\text { to a range of } 2-11 \mathrm{~mm} \\
\text { (mean } 7.0 \mathrm{~mm})(p=0.0002)\end{array}$ \\
\hline $\begin{array}{l}\text { Heinzerling } \\
\text { et al. [20] }\end{array}$ & 2008 & 10 & LL(4) + liver(6) & $4 \mathrm{DCT}$ & $L R, A P, C C+3 D$ & $\begin{array}{l}\text { Mean 3D reduction of } 5.3 \text { and } \\
6.4 \mathrm{~mm} \text { with two compression } \\
\text { levels }(p<0.0001)\end{array}$ \\
\hline $\begin{array}{l}\text { Han } \\
\text { et al. [21] }\end{array}$ & 2010 & 24 & UL(16), ML(2), LL(7) & $4 \mathrm{DCT}$ & LR, AP, CC + 3D & $\begin{array}{l}\text { Mean 3D reduction of } 1.3 \mathrm{~mm} \\
(p<0.05)\end{array}$ \\
\hline $\begin{array}{l}\text { Bengua } \\
\text { et al. [22] }\end{array}$ & 2010 & 19 & UL(11), ML(1), LL(7) & RTRT system & $\mathrm{LR}, \mathrm{AP}, \mathrm{CC}$ & $\begin{array}{l}\text { In CC direction: mean reduction } \\
\text { of } 0.48 \mathrm{~mm} \text { for middle or lower } \\
\text { lobe markers }(p=0.65) \text { and } \\
0.46 \mathrm{~mm} \text { for upper lobe } \\
\text { markers }(p=0.61)^{\mathrm{a}}\end{array}$ \\
\hline
\end{tabular}

Abbreviations: UL, upper lobe; ML, middle lobe; LL, lower lobe; RTRT, real-time radiation therapy; LR, left-right; AP, antero-posterior; CC, cranio-caudal.

${ }^{a}$ Results are given according to lobe tumor location. 
lung receiving a given dose and the dose received by a given volume remained stable. The relative variations that can seem important at first are actually far from absolute variations that would compromise the respect of the lung critical doses. Indeed, SBRT treatments concern small tumors and thus small field sizes. According to RTOG protocols [8,9], three-dimensional custom designed coplanar or non-coplanar fields must be used to deliver highly conformal radiation doses but non-opposing and noncoplanar beams are preferable. Moreover, roughly equallyweighted beams are typically used. In order to be able to compare the two modalities (with and without compression) for all the patients, we systematically used equallydistributed and equally-weighted fields. This way, only the impact of volume modifications induced by the use of abdominal compression is assessed, without being influenced by potential patient-dependent discrepancies in beam arrangements. In most cases, the respect of the lung critical doses can be achieved by adjusting field weights and/or distribution. Our dosimetric results reflect smaller reduction when using abdominal compression than the results presented by Kontrisova et al. [23]. This may be caused by the use of different images to delineate the GTV (3DCT blurred vs. 4DCT MIP), by the use of different CTV to PTV margins, or by the fact that results reported by Kontrisova et al. [23] concern the ipsilateral lung and not the
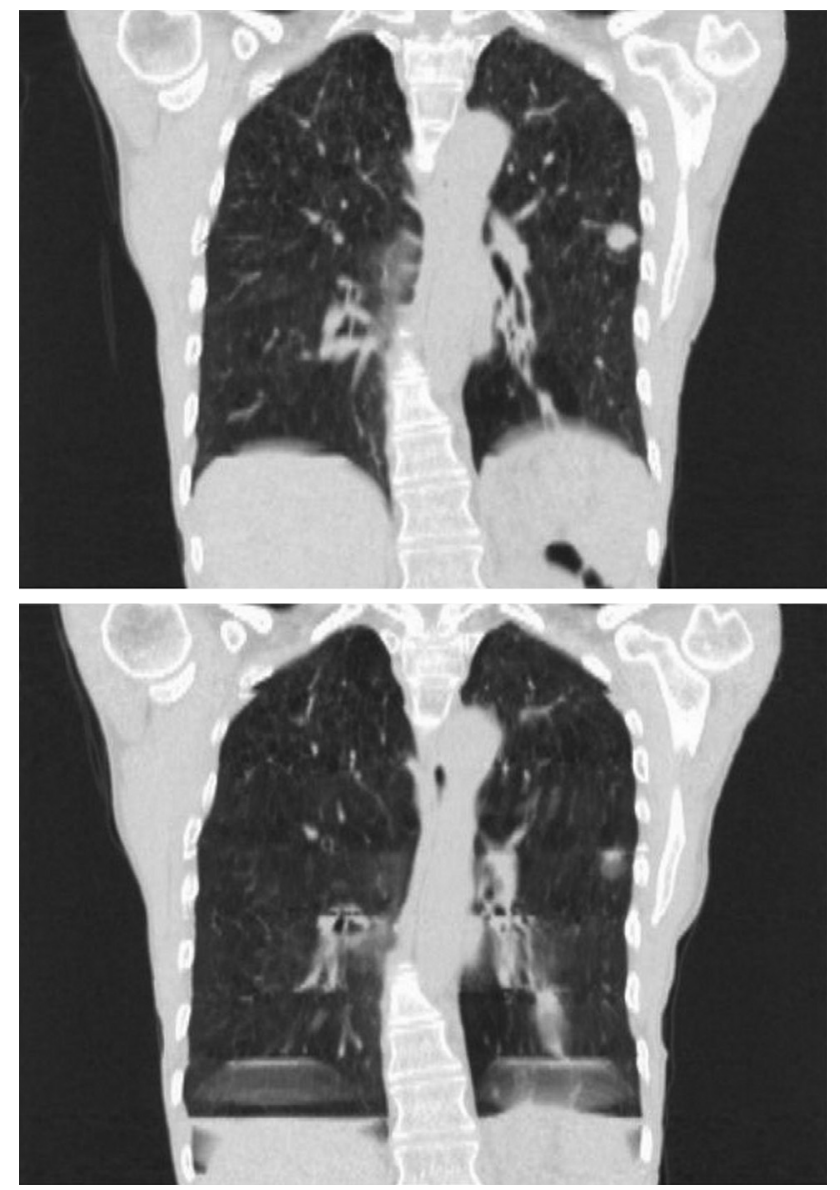

Figure 5 Reduction of motion artifacts with the use of abdominal compression. Slices acquired with (top) and without (bottom) compression. whole healthy lung tissue (left lung + right lung - PTV). A subsample of 4 patients does not allow drawing general conclusions about the efficiency of abdominal compression in reducing healthy lung tissue exposure. However, the chosen patients revealed the highest geometrical gains, illustrating the highest expected dosimetric benefits of the use of compression.

Another consequence of the use of abdominal compression concerns motion artifacts in 4DCT images. Irregularities in the respiratory signal lead to motion artifacts in the reconstructed images. Motion artifacts in 4DCT images reflect interplay between organ motion and scanning movements (gantry rotation and couch displacement). Yamamoto et al. [29] have found that $90 \%$ of the patients have at least one motion artifact in 4DCT images. The endexhale phase is generally the least prone to artifacts because motion is slow and it is reproducible, making it the most stable phase of the breathing cycle [33]. The compression modifies global patient respiration characteristics such as amplitude, frequency or regularity. Consequently, for given acquisition parameters, abdominal compression can limit the occurrence of motion artifacts (Fig. 5) or introduce some. For the patients taking part in this study, we mainly observed an artifact reduction (for 16 patients) whereas artifact increase was less frequent (5 patients).

\section{Conclusions}

The use of abdominal compression for lung SBRT treatments efficiently reduces motion amplitude for lesions close to the diaphragm. If the tumors are large enough, abdominal compression can be associated with an interesting reduction of the internal target volume. For other locations, the use of the compression can provide a minor benefit or even induce unwanted effects such as tumor motion and ITV increase. We have shown that only a small gain in lung healthy tissue sparing could be achieved with the use of abdominal compression.

Based on these findings and taking into account that such a system is time-consuming and not always welltolerated, we have decided to stop using abdominal compression when treating patients with upper and middle lobe tumors. On the contrary, patients with lower lobe lesions still undergo two 4DCT exams, with and without abdominal compression. The relevance of using the compression is evaluated on an individual basis and the decision is taken according to patient tolerance and the expected benefit.

\section{Conflicts of interest statement}

There is no conflict of interest related to the work presented in this paper.

\section{Acknowledgments}

Our work is supported by ETOILE's Research Program (PRRH/UCBL, under CPER 2007-13 funding), without being involved in the study presented in this paper. 


\section{References}

[1] McGarry RC, Song G, des Rosiers P, Timmerman R. Observation-only management of early stage, medically inoperable lung cancer: poor outcome. Chest 2002;121:1155-8.

[2] Nagata Y, Takayama K, Matsuo Y, Norihisa Y, Mizowaki T, Sakamoto T, et al. Clinical outcomes of a phase I/II study of $48 \mathrm{~Gy}$ of stereotactic body radiotherapy in 4 fractions for primary lung cancer using a stereotactic body frame. Int $\mathrm{J}$ Radiat Oncol Biol Phys 2005;63:1427-31.

[3] McGarry RC, Papiez L, Williams M, Whitford T, Timmerman RD. Stereotactic body radiation therapy of early-stage non-smallcell lung carcinoma: phase I study. Int J Radiat Oncol Biol Phys 2005;63:1010-5.

[4] Baumann P, Nyman J, Hoyer M, Wennberg B, Gagliardi G, Lax I , et al. Outcome in a prospective phase II trial of medically inoperable stage I non-small-cell lung cancer patients treated with stereotactic body radiotherapy. J Clin Oncol 2009;27:3290-6.

[5] Timmerman R, Paulus R, Galvin J, Michalski J, Straube W, Bradley J, et al. Stereotactic body radiation therapy for inoperable early stage lung cancer. J Am Med Assoc 2010;303: 1070-6.

[6] Ricardi U, Filippi AR, Guarneri A, Giglioli FR, Ciammella P, Franco $P$, et al. Stereotactic body radiation therapy for early stage non-small cell lung cancer: results of a prospective trial. Lung Cancer 2010;68:72-7.

[7] Hiraoka M, Matsuo Y, Nagata Y. Stereotactic body radiation therapy (SBRT) for early-stage lung cancer. Cancer Radiother 2007;11:32-5.

[8] Timmerman RD, Michalski J, Fowler J, Choy H, Johnstone D, Galvin JM, et al. A phase II trial of stereotactic body radiation therapy (SBRT) in the treatment of patients with medically inoperable stage I/II non-small cell lung cancer. RTOG 0236; 2006.

[9] Videtic GMM, Parker W, Singh AK, Olivier KR, Chang JY, Schild SE, et al. A randomized phase II study comparing 2 stereotactic body radiation therapy (SBRT) schedules for medically inoperable patients with stage I peripheral nonsmall cell lung cancer; 2009. RTOG 0915.

[10] Benedict SH, Yenice KM, Followill D, Hinson W, Kavanagh B, Keall $P$, et al. Stereotactic body radiation therapy: the report of AAPM task group 101. Med Phys 2010;37:4078-101.

[11] Lax I, Blomgren H, Näslund I, Svanström R. Stereotactic radiotherapy of malignancies in the abdomen. Methodological aspects. Acta Oncol 1994;33:677-83.

[12] Blomgren H, Lax I, Näslund I, Svanström R. Stereotactic high dose fraction radiation therapy of extracranial tumors using an accelerator. Clinical experience of the first thirty-one patients. Acta Oncol 1995;34:861-70.

[13] Baba F, Shibamoto Y, Tomita N, Ikeya-Hashizume C, Oda K, Ayakawa $S$, et al. Stereotactic body radiotherapy for stage I lung cancer and small lung metastasis: evaluation of an immobilization system for suppression of respiratory tumor movement and preliminary results. Radiat Oncol 2009;4:15.

[14] Keall PJ, Starkschall G, Shukla H, Forster KM, Ortiz V, Stevens CW, et al. Acquiring $4 \mathrm{D}$ thoracic $\mathrm{CT}$ scans using a multislice helical method. Phys Med Biol 2004;49:2053-67.

[15] Underberg RWM, Lagerwaard FJ, Slotman BJ, Cuijpers JP, Senan S. Use of maximum intensity projections (MIP) for target volume generation in 4DCT scans for lung cancer. Int $J$ Radiat Oncol Biol Phys 2005;63:253-60.

[16] Wulf J, Hädinger U, Oppitz U, Olshausen B, Flentje $M$. Stereotactic radiotherapy of extracranial targets: CTsimulation and accuracy of treatment in the stereotactic body frame. Radiother Oncol 2000;57:225-36.

[17] Keall PJ, Mageras GS, Balter JM, Emery RS, Forster KM, Jiang $\mathrm{SB}$, et al. The management of respiratory motion in radiation oncology report of AAPM task group 76. Med Phys 2006;33:3874-900.

[18] Bissonnette JP, Franks KN, Purdie TG, Moseley DJ, Sonke JJ, Jaffray DA, et al. Quantifying interfraction and intrafraction tumor motion in lung stereotactic body radiotherapy using respiration-correlated cone beam computed tomography. Int J Radiat Oncol Biol Phys 2009;75:688-95.

[19] Negoro Y, Nagata Y, Aoki T, Mizowaki T, Araki N, Takayama K, et al. The effectiveness of an immobilization device in conformal radiotherapy for lung tumor: reduction of respiratory tumor movement and evaluation of the daily setup accuracy. Int J Radiat Oncol Biol Phys 2001;50:889-98.

[20] Heinzerling JH, Anderson JF, Papiez L, Boike T, Chien S, Zhang G, et al. Four-dimensional computed tomography scan analysis of tumor and organ motion at varying levels of abdominal compression during stereotactic treatment of lung and liver. Int J Radiat Oncol Biol Phys 2008;70:1571-8.

[21] Han K, Cheung P, Basran PS, Poon I, Yeung L, Lochray F. A comparison of two immobilization systems for stereotactic body radiation therapy of lung tumors. Radiother Oncol 2010; 95:103-8.

[22] Bengua G, Ishikawa M, Sutherland K, Horita K, Yamazaki R, Fujita $\mathrm{K}$, et al. Evaluation of the effectiveness of the stereotactic body frame in reducing respiratory intrafractional organ motion using the real-time tumor-tracking radiotherapy system. Int J Radiat Oncol Biol Phys 2010;77:630-6.

[23] Kontrisova K, Stock M, Dieckmann K, Bogner J, Pötter R, Georg D. Dosimetric comparison of stereotactic body radiotherapy in different respiration conditions: a modeling study. Radiother Oncol 2006;81:97-104.

[24] Gassa F, Biston MC, Malet C, Lafay F, Ayadi M, Badel JN, et al. The stereotactic body radiation therapy: initiation and clinical program. Cancer Radiother 2006;10:444-50.

[25] Park K, Huang L, Gagne H, Papiez L. Do maximum intensity projection images truly capture tumor motion? Int J Radiat Oncol Biol Phys 2009;73:618-25.

[26] Seroul P, Sarrut D. VV: a viewer for the evaluation of 4D image registration. In: Medical imaging and computer-assisted intervention MICCAI; 2008 [Workshop - System and Architectures for Computer-Assisted Intervention].

[27] Rit S, Pinho R, Delmon V, Pech M, Bouilhol G, Schaerer J, et al. VV, a 4D slicer. In: Medical imaging and computer-assisted intervention miccai 2011; fourth international workshop on pulmonary image analysis; 2011.

[28] Vandemeulebroucke J, Rit S, Kybic J, Clarysse P, Sarrut D. Spatiotemporal motion estimation for respiratory-correlated imaging of the lungs. Med Phys 2011;38:166-78.

[29] Yamamoto T, Langner U, Loo BW, Shen J, Keall PJ. Retrospective analysis of artifacts in four-dimensional CT images of 50 abdominal and thoracic radiotherapy patients. Int J Radiat Oncol Biol Phys 2008;72:1250-8.

[30] Wunderink W, Méndez Romero A, de Kruijf W, de Boer H, Levendag P, Heijmen B. Reduction of respiratory liver tumor motion by abdominal compression in stereotactic body frame, analyzed by tracking fiducial markers implanted in liver. Int $\mathrm{J}$ Radiat Oncol Biol Phys 2008;71:907-15.

[31] Boldea V, Sharp GC, Jiang SB, Sarrut D. 4D-CT lung motion estimation with deformable registration: quantification of motion nonlinearity and hysteresis. Med Phys 2008;35:1008-18.

[32] Seppenwoolde $Y$, Shirato H, Kitamura K, Shimizu S, van Herk $M$, Lebesque JV, et al. Precise and real-time measurement of 3D tumor motion in lung due to breathing and heartbeat, measured during radiotherapy. Int J Radiat Oncol Biol Phys 2002;53:822-34.

[33] Balter JM, Lam KL, McGinn CJ, Lawrence TS, Haken RKT. Improvement of CT-based treatment-planning models of abdominal targets using static exhale imaging. Int J Radiat Oncol Biol Phys 1998;41:939-43. 